\title{
Kinematics of planet-host stars and their relation to dynamical streams in the solar neighbourhood
}

\author{
A. Ecuvillon ${ }^{1}$, G. Israelian ${ }^{1}$, F. Pont ${ }^{2}$, N. C. Santos ${ }^{2,3,4}$, and M. Mayor ${ }^{2}$ \\ 1 Instituto de Astrofísica de Canarias, 38200 La Laguna, Tenerife, Spain \\ e-mail: aecuvill@ll.iac.es \\ 2 Observatoire de Genève, 51 Ch. des Maillettes, 1290 Sauverny, Switzerland \\ 3 Centro de Astronomia e Astrofisica de Universidade de Lisboa, Observatorio Astronomico de Lisboa, Tapada de Ajuda, \\ 1349-018 Lisboa, Portugal \\ 4 Centro de Geofisica de Évora, Rua Romaõ Ramalho 59, 7000 Évora, Portugal
}

Received 21 June 2006 / Accepted 28 August 2006

\section{ABSTRACT}

\begin{abstract}
We present a detailed study on the kinematics of metal-rich stars with and without planets, and their relation to the Hyades, Sirius and Hercules dynamical streams in the solar neighbourhood. Accurate kinematics have been derived for all the stars belonging to the CORALIE planet search survey. We used precise radial velocity measurements and CCF parameters from the CORALIE database, and parallaxes, photometry and proper motions from the HIPPARCOS and Tycho- 2 catalogues. The location of stars with planets in the thin or thick discs has been analysed using both kinematic and chemical constraints. We compare the kinematic behaviour of known planet-host stars to the remaining targets belonging to the volume-limited sample, in particular to its metal-rich population. The high average metallicity of the Hyades stream is confirmed. The planet-host targets show a kinematic behaviour similar to that of the metal-rich comparison subsample, rather than to that of the comparison sample as a whole, thus supporting a primordial origin for the metal excess observed in stars with known planetary companions. According to the scenarios proposed as an explanation for the dynamical streams, systems with giant planets could have formed more easily in metal-rich inner Galactic regions and then been brought into the solar neighbourhood by dynamical streams.
\end{abstract}

Key words. planetary systems - solar neighbourhood - stars: kinematics - stars: abundances - Galaxy: kinematics and dynamics

\section{Introduction}

Precise spectroscopic studies have revealed that stars with planets seem to be particularly metal-rich when compared with "single" field dwarfs (e.g. Gonzalez 1997; Gonzalez et al. 2001; Santos et al. 2001, 2004b, 2005). This represents the first strong link between the planetary companion and its parent star. In the last few years we have published a series of papers on chemical abundances in stars with known giant planets. Abundances of volatile and refractory elements in a large set of planet-host stars and in a volume-limited comparison sample were presented in Ecuvillon et al. (2004a,b, 2006b); Bodaghee et al. (2003); Beirão et al. (2005); Gilli et al. (2006). The volatile/refractory abundance ratios have recently been investigated in Ecuvillon et al. (2006a). Be and Li light elements were also analysed (Santos et al. 2002a, 2004a; Israelian et al. 2004). We are now interested in investigating the kinematic behaviour of planet-host stars.

The kinematics of stars in the solar neighbourhood can give fundamental information for our understanding of the structure and evolution of the Milky Way. ESA's astrometric satellite Hipparcos (ESA 1997) has provided us with accurate positions and trigonometric parallaxes, as well as absolute proper motions, for a large and homogeneous sample of tens of thousands of stars near the Sun. This has offered the opportunity to investigate the velocity distribution in the solar neighbourhood, not only for early-type stars, but also for the old population of the Galactic disc. Several studies have been performed on this topic since (e.g., Dehnen \& Binney 1998; Dehnen 1998;
Alcobé \& Cubarsi 2005; Chereul et al. 1999; Hoogerwerf \& Aguilar 1999; Asiain et al. 1999; Mignard 2000).

The kinematic distribution of stars in the Solar neighbourhood is far from smooth. The inhomogeneities can be spatially confined groups of young stars (e.g. young clusters), or spatially extended groups with the same kinematics. Eggen (1994) defined a "supercluster" (SC) as a group of gravitationally unbound stars that share the same kinematics and may occupy extended regions in the Galaxy, and a "moving group" (MG) as the part of the supercluster that enters the solar neighbourhood and can be observed all over the sky.

According to the standard interpretation, stars in a moving group were formed simultaneously in a small phase-space volume, so that we can still observe a stream of young stars with similar velocities. On the other hand, old stellar populations should be completely mixed and present a smooth distribution function, due to phase mixing and scattering processes. There are two factors acting against the persistence of a moving group in the general stellar background: Galactic differential rotation, which tends to spread them out very quickly in the direction of the Galactic rotation (Woolley 1960), and disc heating (e.g. Lacey 1991), which produces an increase in the velocity dispersion of disc stars with age. Wielen (1977) reported that a typical star in the solar neighborhood would lose trace of its initial peculiar velocity within $2 \times 10^{8}$ years.

However, several works have obtained results that raise problems with this interpretation. On the one hand, it has been reported that early-type stars belonging to the same supercluster 
span a wide range of ages, in contradiction with the assumption of a common origin (Chereul et al. 1998, 1999). On the other hand, some of the classical MGs are several $10^{8} \mathrm{yr}$ old, and that significant clumpiness in velocity space has been reported for late-type stars (Dehnen 1998). Other mechanisms of a different nature have been proposed as responsible for the substructures observed in velocity space. Streams could be the remnants of merger events between the Milky Way and a satellite galaxy, as recently proposed by Navarro et al. (2004) for the Arcturus group, although the chance that two of them have left such important signature in the disc near the Sun is statistically very low (Famaey et al. 2005a). Raboud et al. (1998) reported that the local anomaly found in the $(u, v)$ plane is caused by intermediateto high-metallicity stars, while a merger would involve rather metal-poor stars (Dehnen 2000).

Another possibility is that purely dynamical mechanisms, such as non-axysimmetric components of the gravitational potential (for example, the rotating galactic bar or spiral waves), are the cause of substructures in the velocity distribution (e.g. Dehnen 1998; Raboud et al. 1998; Sellwood \& Binney 2002). Stars in an MG could have formed in the inner part of the disc and have become trapped in resonant orbits. Dehnen (1999) associates the bimodality of the local velocity distribution of late-type disc stars, lately identified as the Hercules stream, with high-metallicity stars thrown out from the inner disc, and Dehnen (2000) concluded that outer Lindblad resonant scattering off the Galactic bar is presently the only viable explanation for it. Other simulations have tested the effect of other nonaxysimmetric components, in particular transient spiral waves, in the Galactic disc (e.g. Sellwood \& Binney 2002; De Simone et al. 2004), with the result that they may cause radial migration near their corotation radius.

It is not unlikely that known exoplanetary systems could also have suffered such radial displacements. The typical contributors to the kinematic structures in the solar neighbourhood are metalrich old stars (Raboud et al. 1998). The well-known metal-rich nature of planet-host stars (e.g. Gonzalez 1997; Gonzalez et al. 2001; Santos et al. 2001, 2004b, 2005) might thus be related to dynamical streams. It is probable that planet-host stars may have formed in protoplanetary clouds with a high metal content, such as those located in inner regions of the Milky Way, and then be brought closer to the Sun by dynamical streams (Famaey et al. 2005b). Famaey et al. (2005b) have found an indicative clump of stars hosting planets from Santos et al. (2003) in the Hyades streams. Several works have investigated the kinematics of stars with extrasolar planets (e.g. Gonzalez 1999; Reid 2002; Barbieri \& Gratton 2002; Santos et al. 2003), without finding any significative peculiarity.

Two main scenarios have been proposed to explain the metal excess found in stars with exoplanets. The "self-enrichment" hypothesis suggests that the infall of a large amount of protoplanetary rocky material on to the parent star may have enhanced its primordial metallicity (Gonzalez 1997; Laughlin 2000). In contrast, Santos et al. $(2000,2001)$ have proposed a "primordial" origin for the iron overabundance of planet-host stars, so that planetary system formation would be triggered by a high metal content of the primordial cloud. Several works have obtained results supporting the "primordial" scenario (Pinsonneault et al. 2001; Santos et al. 2004b; Reid 2002; Sadakane et al. 2002; Ecuvillon et al. 2004a,b, 2006b,a; Gilli et al. 2006). However, some clear evidence of pollution events have been reported (Gonzalez 1998; Laws \& Gonzalez 2001; Smith et al. 2001; Israelian et al. 2001, 2003), so that the "self-enrichment" scenario cannot be completely discarded (Vauclair 2004).
This paper studies the kinematics of planet-host stars and their relation to the dynamical streams in the solar neighbourhood and investigates whether it corresponds to the behaviour of the metal-rich population or not. If the metal-rich nature of planet-host stars is intrinsic, then they should be kinematically identical to the metal-rich comparison sample, while if the metallicity excess is due to planet-related enrichment, then they should be kinematically identical to the total comparison sample.

We present accurate kinematics for all the stars belonging to the CORALIE planet search survey (see Udry et al. 2000). Precise radial velocity measurements and CCF parameters were extracted from this unique database, whereas HIPPARCOS (ESA 1997) parallaxes and photometry, and Tycho-2 (Høg et al. 2000) proper motions were adopted. The location of stars with planets in the thin or thick discs is analysed using both kinematic and chemical constraints. We study the kinematic behaviour of known planet-host targets with respect to the remaining stars belonging to the kinematically unbiased volume-limited sample and we investigate possible links between the presence of planets, the stellar metallicity and the dynamical streams Hyades, Sirius and Hercules.

\section{Data and analysis}

We obtained kinematics for all the stars belonging to the CORALIE planet search survey. The overall sample consists of about 1650 unevolved solar-type stars closer than $50 \mathrm{pc}$, with precise parallaxes $\left(\sigma_{\Pi} \leq 5 \mathrm{mas}\right)$ and spectral types between $\mathrm{F} 8$ and M1 in the HIPPARCOS catalogue. A further selection was applied to exclude binaries $\left(\sigma_{R V}<500 \mathrm{~m} \mathrm{~s}^{-1}\right)$. We used parallax measurements and photometric data from the HIPPARCOS catalogue (ESA 1997), and coordinates and stellar motions were extracted from Tycho-2 catalogue (Høg et al. 2000), since they improved the HIPPARCOS data by combining several new catalogues. The CORALIE database provided us with radial velocity measurements of a unique precision, as well as with the average width and depth of the cross correlation function (CCF). We derived Galactic space-velocity components $U, V$ and $W$ with respect to the Local Standard of Rest (LSR), adopting the standard solar motion $\left(U_{\odot}, V_{\odot}, W_{\odot}\right)=(10.00,5.25,7.17) \mathrm{km} \mathrm{s}^{-1}$ from Dehnen \& Binney (1998).

\subsection{Thin/thick disc}

We selected thin and thick disc stars from our sample following the procedure described by Bensby et al. (2003). We assumed that the Galactic space velocities of each population (thin disc, thick disc and halo stars) have Gaussian distributions. The characteristic velocity dispersions $\left(\sigma_{U}, \sigma_{V}, \sigma_{W}\right)$ and asymmetric drift $\left(V_{\text {asym }}\right)$ were taken from Bensby et al. (2003), as well as the observed fractions of each population in the solar neighbourhood $(X)$. The probability distribution of belonging to a specific population $(P)$ is thus given by the Gaussian probability distribution multiplied by the corresponding observed fraction $(X)$. Stars with $P($ thick disc $) / P($ thin disc $) \geq 10$ and $\leq 0.1$ will be reliable members of the thick and thin disc, respectively. Figure 1 shows the probability distributions of belonging to the thin disc (upper panel) and to the thick disc (central panel), and the thick-discto-thin-disc relative probability (lower panel). Chemical abundances for CORALIE stars already spectroscopically studied were extracted from Ecuvillon et al. (2004a,b, 2006b) and Gilli et al. (2006) and combined with the kinematic information in order to analyse the relation of the trends $[X / \mathrm{Fe}]$ vs. $[\mathrm{Fe} / \mathrm{H}]$ with the thin and thick discs. 


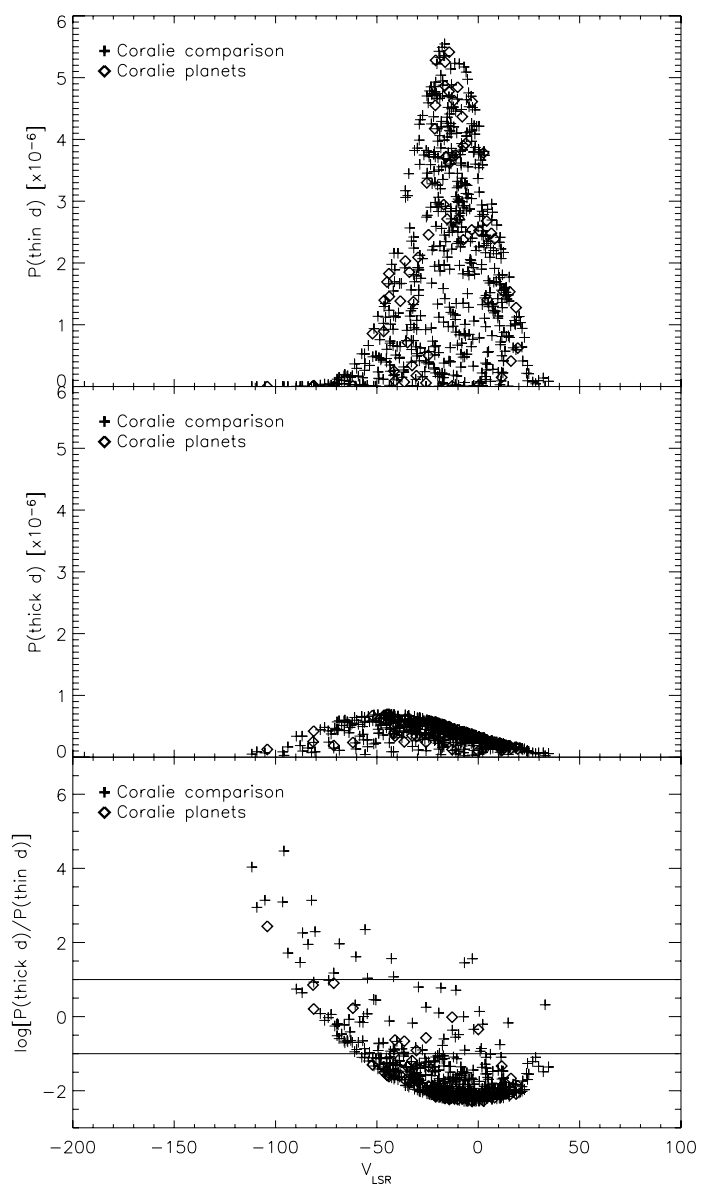

Fig. 1. Probability distributions of location in the thin disc (upper panel) and the thick disc (central panel), and the thick-disc-to-thin-disc relative probability (lower panel). Diamonds and crosses represent stars with and without planets, respectively. In the lower panel, the solid lines indicate the limits for reliable membership to each population.

All the metallicities were derived using the calibration of the CCF surface with $[\mathrm{Fe} / \mathrm{H}]$ by Santos et al. (2002b). The distribution of the thick-disc-to-thin-disc relative probability as a function of metallicity is shown in Fig. 2. We also computed stellar masses by a comparison of the star positions in the CM diagram with the calculated isochrones of Pietrinferni et al. (2004). Ages for field stars are notoriously difficult to determine, and suffer from large biases that make them unsuitable for this kind of study, as discussed by Pont \& Eyer (2004). However, mass can be used as an age surrogate for a first-order separation of young and old stars. Masses can be determined quite precisely from stellar evolution models and are less subject to biases. Figure 3 shows the metallicity distributions for planet-host and comparison stars (upper panel), and the plot of $[\mathrm{Fe} / \mathrm{H}]$ vs. mass (lower panel).

\subsection{Analysis of the $(U, V, W)$ space}

The field ellipsoid was defined by computing its inertial tensor: the eigenvectors of the inertial tensor would indicate the main directions $\left(U^{\prime}, V^{\prime}, W^{\prime}\right)$ of the field ellipsoid in the $(U, V, W)$ space. We adopted $2 \sigma$ of the data along the directions $U^{\prime}, V^{\prime}$ and $W^{\prime}$ as the field ellipsoid semiaxes. We then derived the density distribution of the Galactic space velocities by the fifth nearest-neighbour method. This method assigns to each point a

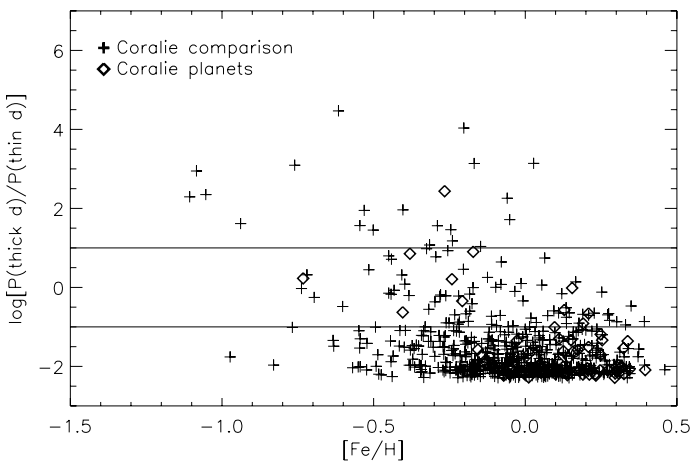

Fig. 2. Thick-disc-to-thin-disc relative probability as a function of $[\mathrm{Fe} / \mathrm{H}]$. Diamonds and crosses represent stars with and without planets, respectively. The solid lines indicate the limits for the reliable membership to each population.

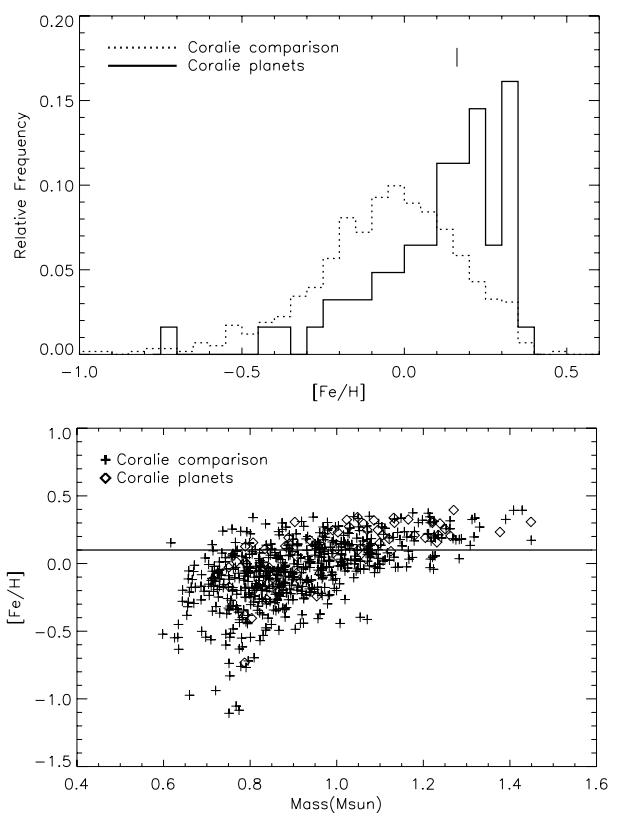

Fig. 3. Upper panel: metallicity distributions of planet-host and comparison stars (solid and dotted lines, respectively). The mark indicates the median of the planet-host distribution. Lower panel: $[\mathrm{Fe} / \mathrm{H}]$ vs. stellar mass (in solar mass units) for planet-host (diamonds) and comparison (crosses) stars.

density value, according to its surroundings (see Silverman 1996, Sect. 5.2).

A field without "structures" is supposed to follow a Gaussian distribution. Therefore, this Gaussian "background" has to be removed to study the remnant "structures". We thus subtracted from the field density distribution the density distribution of the Gaussian corresponding to the previously defined field ellipsoid. Figures 4-6 illustrate the density distribution of Galactic space velocities projected on the $(V, U),(W, V)$ and $(W, U)$ planes, respectively. The uncorrected distributions are shown in the upper panels, while the subtracted Gaussian distributions and the corrected density distributions are presented in the lower panels.

The next step consisted in defining the ellipsoids describing the remnant "structures" in the corrected density distribution. As a first aproximation, we adopted the kinematic parameters of the Hyades, Sirius and Hercules streams derived by Famaey et al. (2005a), and then adjusted by eye the ellipsoid centroids and semiaxes according to what we observed. Table 1 lists the kinematic parameters finally adopted for each velocity ellipsoid. 


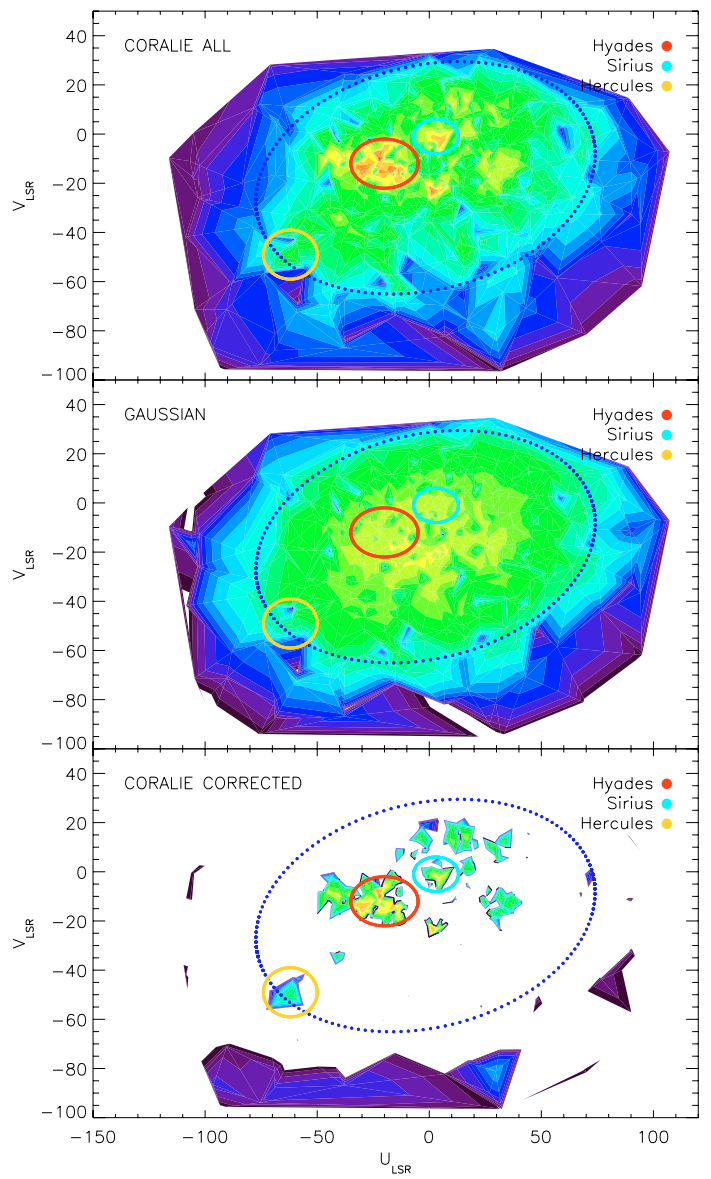

Fig. 4. Density distribution of Galactic space velocities projected on the $(V, U)$ plane, for the whole comparison sample. The uncorrected density distribution, the subtracted Gaussian distribution and the corrected density distribution are presented in the upper, central and lower panels, respectively. The field ellipsoid is overplotted with the blue line, while the Hyades, Sirius and Hercules ellipsoids are indicated by the red, blue green and yellow lines.

In Figs. 4-6 the projected ellipsoids are represented and identified as the Hyades, Sirius and Hercules streams. The projected field ellipsoid is also overplotted in each figure (blue line). The number of targets falling into each velocity ellipsoid are listed in Table 2.

The method used to obtain the field ellipsoid does not make any a priori assumptions concerning the dynamical streams. A different approach would be to adopt a negligible vertex deviation for the field ellispoid by considering that the Hercules stream is its main contributor (e.g. Mühlbauer \& Dehnen 2003). We have checked that the assumption of a negligible vertex deviation does not change our results. Although the Hercules ellipsoid results slightly enlarged, and consequently the number of comparison stars belonging to the stream is enhanced from 5 to 8 , no planet-host stars are found within the modified Hercules ellipsoid.

Next, we defined the groups in which our sample would be divided for the comparison: planet hosts, comparison sample, metal-rich comparison stars and comparison stars with solar metallicity or lower. The metallicity cutoff was chosen so that the median of the metal-rich comparison sample was equal to the one of the planet-host distribution $([\mathrm{Fe} / \mathrm{H}]=0.15$, see Fig. 3, upper panel), and also so that the mass-metallicity, or age-metallicity, relation affected as few targets as possible (see

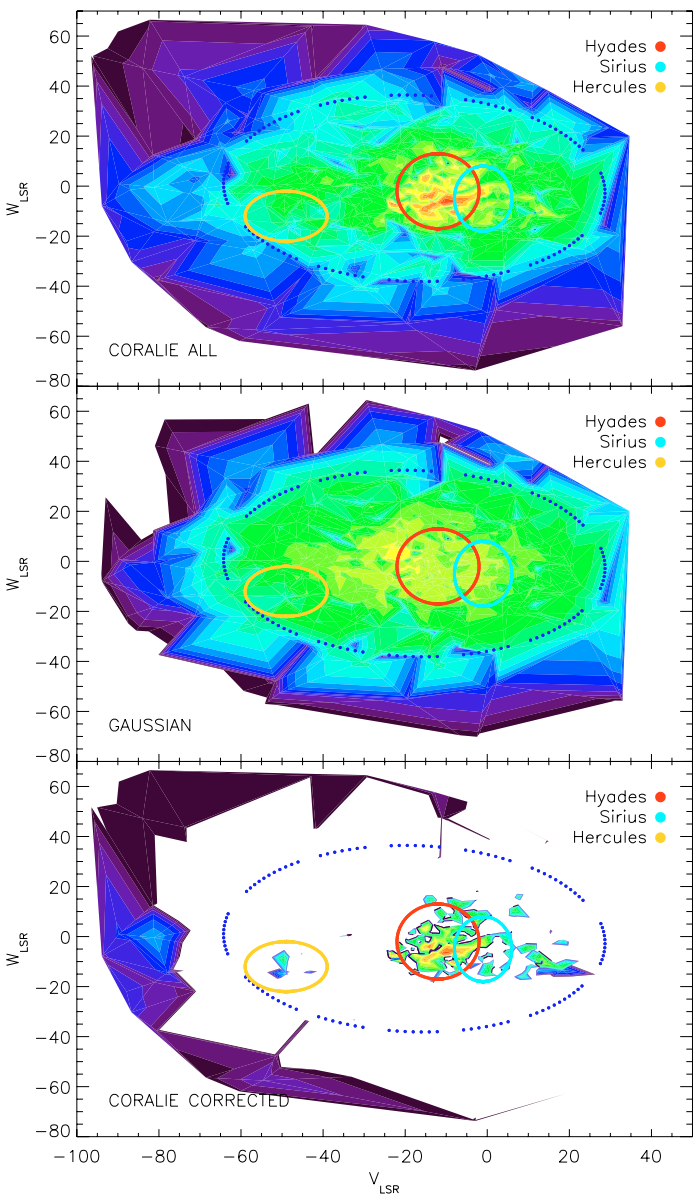

Fig. 5. Density distribution of Galactic space velocities projected on the $(W, V)$ plane for the whole comparison sample. The uncorrected density distribution, the subtracted Gaussian distribution and the corrected density distribution are presented in the upper, central and lower panels, respectively. The field ellipsoid is overplotted with the blue line, while the Hyades, Sirius and Hercules ellipsoids are indicated by the red, blue green and yellow lines.

Fig. 3, lower panel). Cutting the comparison sample at $[\mathrm{Fe} / \mathrm{H}]=$ 0.07 fulfilled the condition concerning medians. The metallicity cutoff was then rounded off to $[\mathrm{Fe} / \mathrm{H}]=0.1$.

We then count how many stars from each group fell into the ellipsoids corresponding to the field and to the Hyades, Sirius and Hercules streams. We also counted the numbers expected in the streams if the group were to have followed a Gaussian distribution, with $\sigma$ equal to the velocity dispersion of the group. For the planet-host sample, we did this for two Gaussian distributions, the first with the dispersion characteristic of the whole comparison sample, and the second with the dispersion characteristic of the metal-rich comparison subsample. The count for each stream was corrected by subtracting the expected number from the Gaussian distribution and normalized by dividing by the total number of the group in the field. This corresponds to the ratio of excess objects in the stream. The Sirius and Hercules streams were excluded from this analysis since the two velocity ellipsoids contained respectively one and no planet host stars.

A further analysis was carried out by means of the density distributions of the Galactic space velocities, derived by using the fifth nearest-neighbour method. For each group independently, we computed the density distribution corresponding to a Gaussian distribution with $\sigma$ equal to the velocity dispersion of the group. In the case of the planet-host group, two Gaussian 


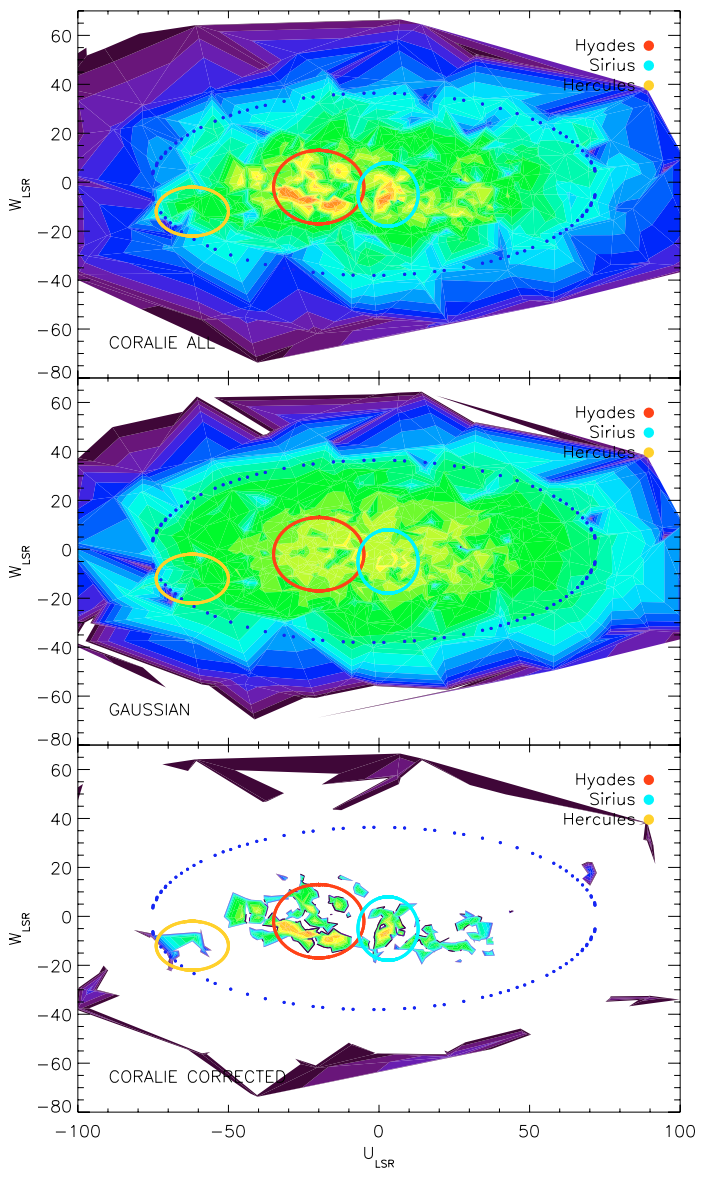

Fig. 6. Density distribution of Galactic space velocities projected on the $(W, U)$ plane for the whole comparison sample. The uncorrected density distribution, the subtracted Gaussian distribution and the corrected density distribution are presented in the upper, central and lower panels, respectively. The field ellipsoid is overplotted with the blue line, while the Hyades, Sirius and Hercules ellipsoids are indicated by the red, blue green and yellow lines.

Table 1. Kinematic parameters adopted for the Hyades, Sirius and Hercules velocity ellipsoids. The mean velocities $\left(U_{0}, V_{0}, W_{0}\right)$ are relative to the LSR. All values are expressed in $\mathrm{km} \mathrm{s}^{-1}$.

\begin{tabular}{c|rrr}
\hline \hline$\left(\mathrm{km} \mathrm{s}^{-1}\right)$ & Hyades & Sirius & Hercules \\
\hline$U_{0}$ & -20 & 3 & -62 \\
$U$ semiaxis & 15 & 10 & 12 \\
$V_{0}$ & -12 & -1 & -49 \\
$V$ semiaxis & 10 & 7 & 10 \\
$W_{0}$ & -2 & -5 & -12 \\
$W$ semiaxis & 15 & 13 & 10 \\
\hline
\end{tabular}

distributions were tested, one with the dispersion of the whole comparison sample, and the other with the dispersion of the metal-rich comparison subsample. The "observed" distribution was then corrected by the Gaussian contribution. From the corrected density distribution, we derived an average density for the field and the Hyades ellipsoids. The averaged field density was then subtracted that of the Hyades in order to obtain the overdensity over the mean field "level".
Table 2. Number of targets falling into the velocity ellipsoids adopted for the Hyades, Sirius and Hercules streams.

\begin{tabular}{lcccc}
\hline \hline & Field & Hyades & Sirius & Hercules \\
\hline Comparison stars & 440 & 40 & 16 & 5 \\
Planet-host stars & 47 & 10 & 1 & 0 \\
\hline
\end{tabular}

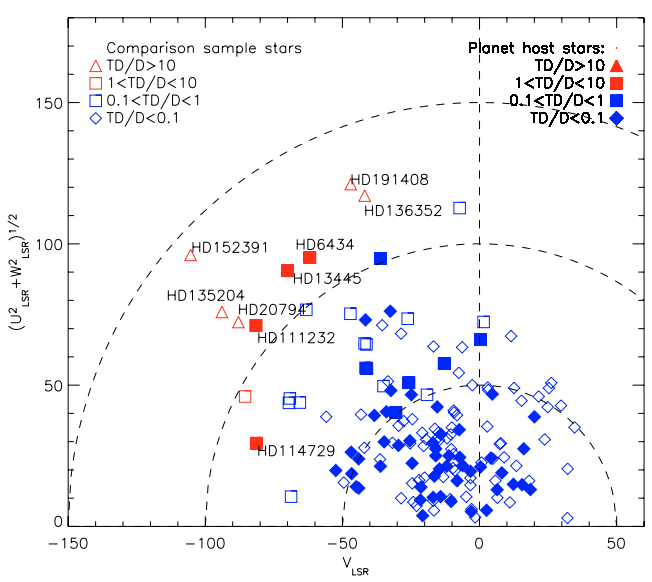

Fig. 7. Toomre diagram for the CORALIE targets with chemical abundances by Ecuvillon et al. (2004a,b, 2006b) and Gilli et al. (2006). Dotted lines indicate constant peculiar velocities $v_{\text {pec }}=\left(U_{\mathrm{LSR}}^{2}+V_{\mathrm{LSR}}^{2}+\right.$ $\left.W_{\mathrm{LSR}}^{2}\right)^{1 / 2}$ in steps of $50 \mathrm{~km} \mathrm{~s}^{-1}$. Filled and open symbols represent stars with and without planets, respectively. Targets that are full and intermediate members of the thick disc population are indicated with red triangles and squares, respectively, while full and intermediate members of the thin disc are represented by blue diamonds and squares, respectively. The four comparison targets and the four planet-host stars of the thick disc are identified by their numbers.

\section{Results and discussion}

\subsection{Thin/thick disc}

Our results reveal that the CORALIE sample has one planet-host target, HD 4308, and 21 stars with no known planetary companions, with kinematics typical of a thick disc (see Fig. 1). The metallicities of HD $4308([\mathrm{Fe} / \mathrm{H}]=-0.27$ from the $\mathrm{CCF}$ calibration) and of the thick disc targets without known planets are typical of the thick disc population (see Fig. 2). Only one comparison star, HD 152391, shows a supersolar metallicity ( $[\mathrm{Fe} / \mathrm{H}]=0.03$ from the CCF calibration). The following study combining both kinematical and chemical information will be limited to the subset of CORALIE stars with available homogeneous abundances from Ecuvillon et al. (2004a,b, 2006b) and Gilli et al. (2006).

The Toomre diagram for the subset of CORALIE stars with chemical abundances from Ecuvillon et al. (2004a,b, 2006b) and Gilli et al. (2006) is shown in Fig. 7. Five comparison stars are very likely members of the thick disc population (HD 20794, HD 135204, HD 136352, HD 152391, HD 191408), while no planet-host targets present such a high probability. Only the four planet hosts HD 6434, HD 13445, HD 111232 and HD 114729, are potential members of the thick disc, with probabilities $P($ thick disc $) / P($ thin disc) between 1 and 10 . The $[X / \mathrm{Fe}]$ vs. $[\mathrm{Fe} / \mathrm{H}]$ trends for oxygen and magnesium are shown in Fig. 8 , and in Figs. 9 and 10 for the rest of the elements (C, N, Na, Al, $\mathrm{Si}, \mathrm{S}, \mathrm{Ca}, \mathrm{Sc}, \mathrm{Ti}, \mathrm{V}, \mathrm{Cr}, \mathrm{Mn}, \mathrm{Co}, \mathrm{Ni}, \mathrm{Cu}$ and $\mathrm{Zn}$ ).

Previous works (e.g. Fuhrmann 1998; Feltzing et al. 2003; Bensby et al. 2003) reported different abundance trends for the thin and thick disc populations. In particular, the $\alpha$-elements 

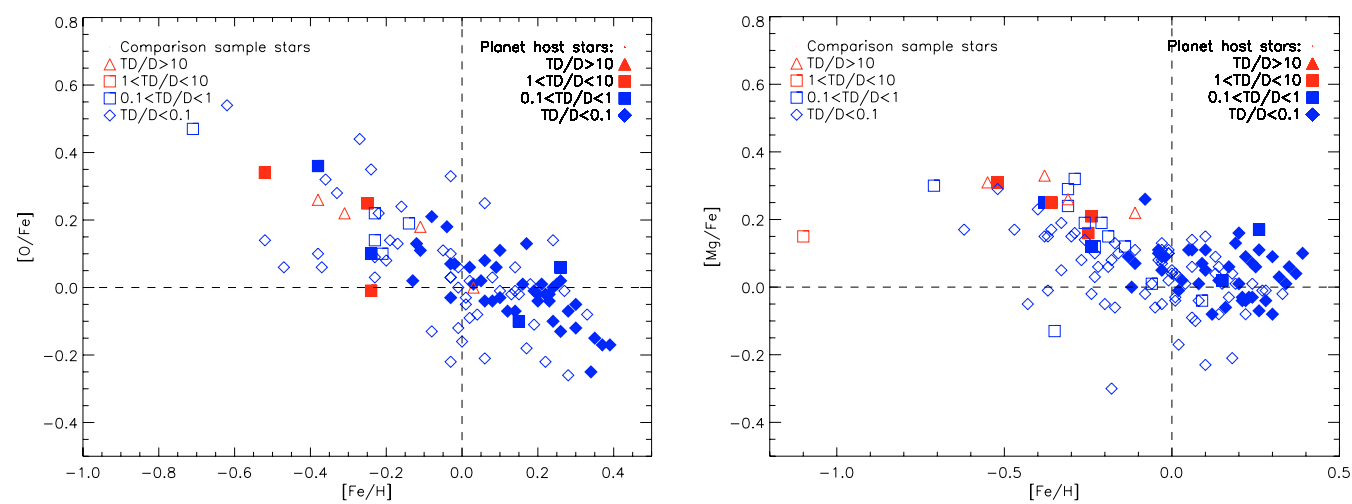

Fig. 8. $[\mathrm{O} / \mathrm{Fe}]$ and $[\mathrm{Mg} / \mathrm{Fe}]$ vs. $[\mathrm{Fe} / \mathrm{H}]$ for CORALIE targets with chemical abundances by Ecuvillon et al. (2004a,b, 2006b) and Gilli et al. (2006). Filled and open symbols represent stars with and without planets, respectively. Targets which are full and intermediate members of the thick disc population are indicated with red triangles and squares, respectively, while full and intermediate members of the thin disc are represented by blue diamonds and squares, respectively.

oxygen and magnesium show clearly distinct trends for the thin and thick discs at sub-solar metallicities (Bensby et al. 2003, 2004). However, Chen et al. (2000) presented results from a sample of $90 \mathrm{~F}$ and $\mathrm{G}$ dwarfs which show no significant scatter in $\alpha$-element ratios as a function of $[\mathrm{Fe} / \mathrm{H}]$. Another interesting issue is the existence of stars with thick disc kinematics at high metallicities, pointed out by several authors (e.g. Bensby et al. 2003; Mishenina et al. 2004).

Our results confirm the possibility of thick disc stars at $[\mathrm{Fe} / \mathrm{H}]>0$, since one of the five comparison stars with thick disc kinematics that was previously chemically analysed, HD 152391, has $[\mathrm{Fe} / \mathrm{H}]=0.03$. The same target has been already reported as a thick disc member with supersolar metallicity in the kinematic study of the whole CORALIE sample (see the first paragraph of this section). It is interesting to note how the metallicity derived with the CCF calibration agrees perfectly with the iron abundance from the detailed spectroscopic study by Santos et al. (2005).

Nevertheless, we did not find any difference in the abundance patterns marked by thick and thin disc stars, nor any significant signature of enrichment in $\alpha$-elements for thick disc population. For magnesium (see Fig. 8, right panel), it might be possible that the thick disc members lie on the upper envelope of the points. However, the small number of possible members for this population in our sample does not allow us to reach any conclusion.

\subsection{Dynamical streams}

Table 3 shows the results obtained with the "counting" method, using the ellipsoid definitions from our work (Definition 1), and the ones from Famaey et al. (2005b) (Definition 2). The first column lists the different groups: planet-host stars, assuming for the Gaussian the $\sigma$ of the whole comparison sample, and of the metal-rich subsample; the whole comparison sample; and the metal-rich and the solar-metallicity comparison subsamples. Then are listed the numbers of targets in the field and Hyades ellipsoids, the expected number of targets from the Gaussian distribution, and finally the ratio of objects in excess from what expected for a Gaussian distribution. The uncertainties were estimated as Poissonian errors.

The results from this method indicate that the clumpiness typical of the Hyades is much more significant for the metalrich subsample than for the whole sample. This confirms the high average metallicity reported for Hyades stream members by previous studies (e.g. Raboud et al. 1998; Chereul \& Grenon 2001) and agrees with the mechanism proposed as an explanation of the anomalies in the $(U, V)$ plane (Dehnen 2000; Famaey et al. 2005a). According to this description, the stars would have formed at inner Galactic radii, with higher metallicity, and then be trapped into resonant orbits by the non-axysimmetric potential, and thrown closer to the Sun.

The planet-host group shows an excess in the Hyades stream much more significant than the other groups. Errors associated with stars with planets are large because of the small number of targets with planetary companions involved in the whole volume-limited sample of CORALIE. However, the targets with planets present an excess in the Hyades stream significantly larger than that obtained for the other groups. In particular, the clumpiness of planet-host stars is different from that of the whole comparison sample, with a significance of more than $1.5 \sigma$.

The kinematic behaviour of CORALIE stars with planets seems to match better with that observed in the metal-rich comparison subsample than with the whole comparison sample. Both metal-rich and planet-host samples show a much larger clumpiness than the whole comparison sample. Nevertheless, the presence of planet-host stars in the Hyades stream is even denser than that found for metal-rich "single" stars. This outcome is not altered by changing the assumption of the characteristic velocity dispersion of the group with planets; there is no significant difference in adopting a Gaussian distribution with the $\sigma$ of the whole comparison sample, or of the metal-rich subsample.

If the kinematic parameters of dynamical streams by Famaey et al. (2005a) are adopted (see Table 3, Definition 2), the results are similar to those reported above. The differences between the excesses for the comparison groups obtained using the two kinematic parameter sets are mostly irrelevant. For planet-host stars, the two definitions give larger discrepancies. In the Definition 2 case, the group of stars with planets behaves in the same way as the metal-rich comparison subsample. However, the analysis by Famaey et al. (2005a) involved K and M giants, while the targets of the volume-limited CORALIE survey are mostly G and $\mathrm{K}$ dwarfs. In that sense, the determination of the dynamical stream parameters could be affected by the target spectral type. The kinematic parameters of the dynamical streams obtained in our work are thus considered more suitable for our analysis.

The results from the "overdensity" method are listed in Table 4. The Def. 1 column refers to the case adopting the kinematic parameters of the streams ellipsoids from our work and $[\mathrm{Fe} / \mathrm{H}]=0.1$ as metallicity cutoff, and subtracting the averaged 

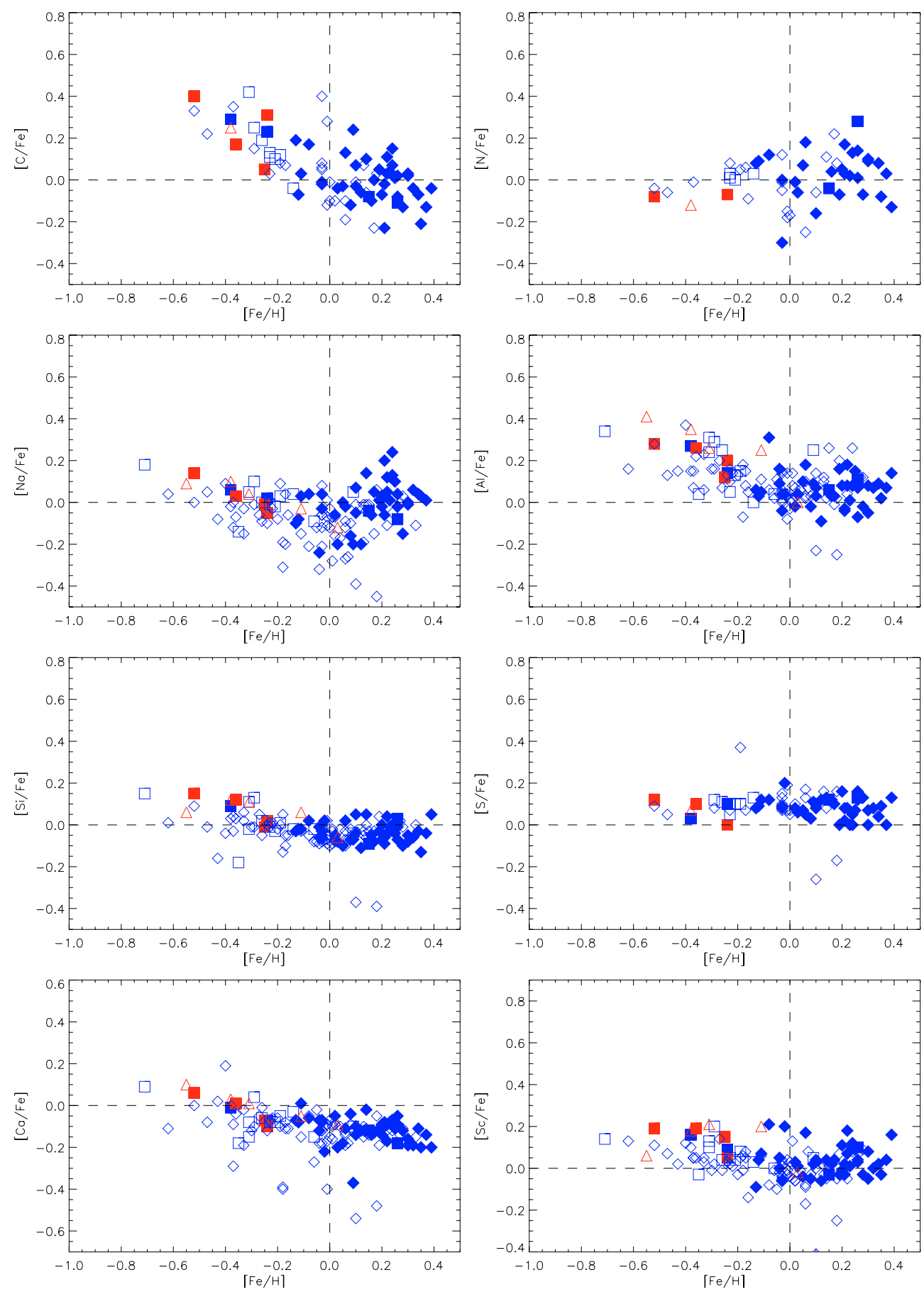

Fig. 9. $[X / \mathrm{Fe}]$ vs. $[\mathrm{Fe} / \mathrm{H}]$ (from $\mathrm{C}$ to $\mathrm{Sc}$ ) for CORALIE targets with chemical abundances by Ecuvillon et al. (2004a,b, 2006b) and Gilli et al. (2006). Filled and open symbols represent stars with and without planets, respectively. Targets which are full and intermediate members of the thick disc population are indicated with red triangles and squares, respectively, while full and intermediate members of the thin disc are represented by blue diamonds and squares, respectively.

Table 3. Results for the "counting" method in the Hyades stream. The Definition 1 and Definition 2 columns refer to the adopted definitions for the stream ellipsoids, from our work and from Famaey et al. (2005a), respectively. The numbers of stars lying in the field and Hyades ellispoids are listed, as well as the expected number of stars for a Gaussian distribution, and the resulting ratio of objects in excess in the Hyades stream. The assumed uncertainties are Poissonian errors.

\begin{tabular}{c|cccc|cccc}
\hline \hline & \multicolumn{4}{|c|}{ Definition 1 } & \multicolumn{4}{c}{ Definition 2 } \\
Group description & Field & Hyades & Exp Hyades & Excess & Field & Hyades & Exp Hyades & Excess \\
\hline Planet-hosts: $\sigma$ whole comp. & 47 & 10 & 1.7 & $0.18 \pm 0.07$ & 47 & 7 & 1.4 & $0.12 \pm 0.05$ \\
$\sigma$ M-rich comp. & $\prime$ & $\prime$ & 2.3 & $0.16 \pm 0.06$ & $\prime$ & $\prime$ & 1.9 & $0.11 \pm 0.06$ \\
Whole comparison & 440 & 40 & 16.3 & $0.05 \pm 0.01$ & 440 & 33 & 13.0 & $0.05 \pm 0.01$ \\
M-Rich comparison & 119 & 19 & 5.7 & $0.11 \pm 0.04$ & 119 & 17 & 4.7 & $0.10 \pm 0.03$ \\
Solar comparison & 321 & 21 & 11.9 & $0.03 \pm 0.02$ & 321 & 16 & 9.2 & $0.02 \pm 0.01$ \\
\hline
\end{tabular}



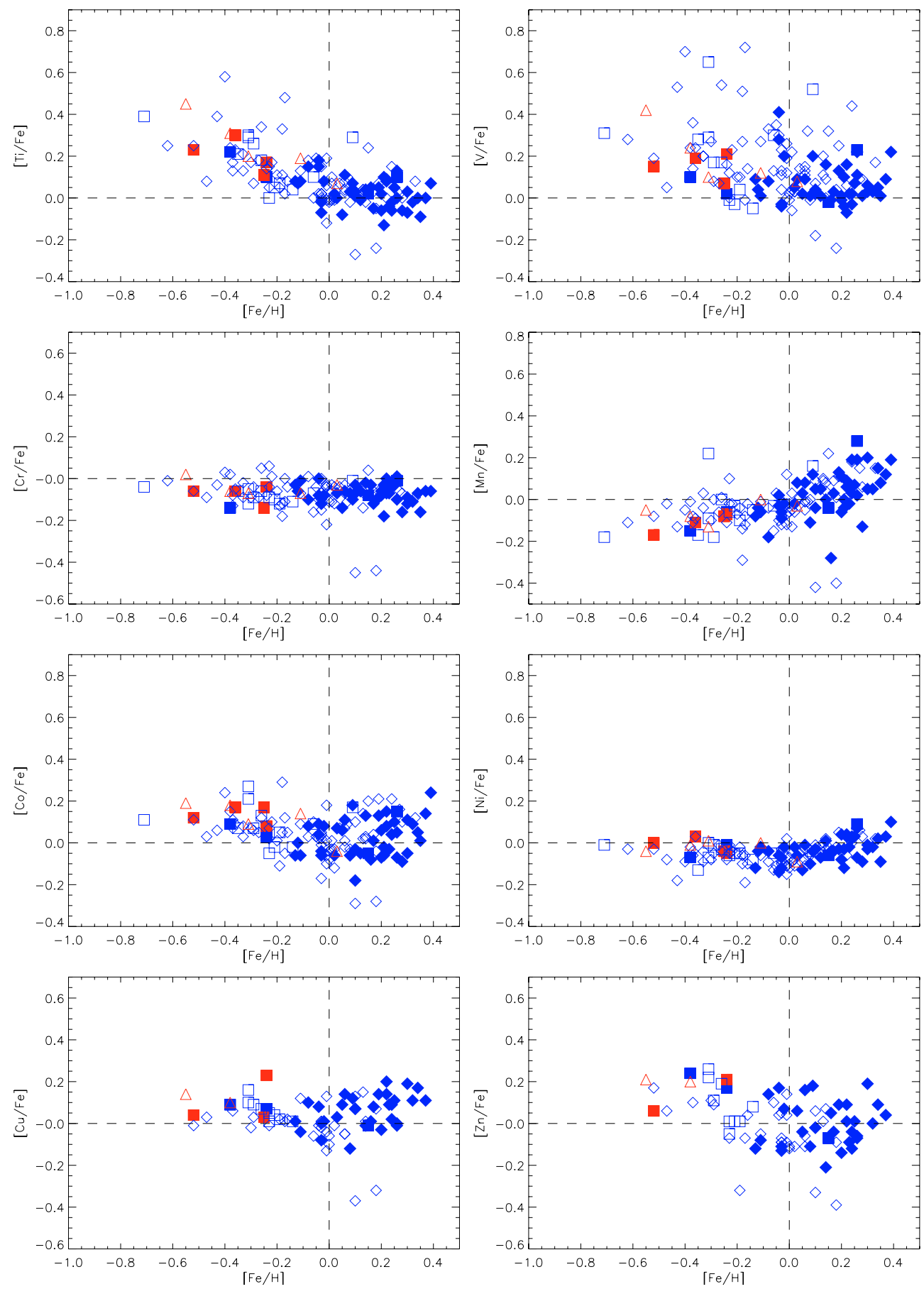

Fig. 10. Continuation of Fig. 9. From Ti to Zn.

Table 4. Results for the "overdensity" method in the Hyades stream. The Def. 1 and Def. 2 columns refer to the adopted definitions for the velocity ellipsoids, from our work and from Famaey et al. (2005a), respectively. Def. $1 \mathrm{mod}$. 1 corresponds to the case without substracting the average density of the field, while Def. $1 \mathrm{mod}$. 2 reports the results for a metallicity cutoff $[\mathrm{Fe} / \mathrm{H}]_{\text {cutoff }}=0.05$ (see the Sect. 3.2 for more details). The last column, Def. $1 M<1.2 M_{\odot}$, refers to the results obtained by excluding the targets with $M>1.2 M_{\odot}$. The uncertainties were derived applying the bootstrap method.

\begin{tabular}{c|c|c|c|c|c}
\hline \hline & \multicolumn{5}{|c}{ Hyades overdensity } \\
Group description & Def. 1 & Def. 2 & Def. 1 mod. 1 & Def. 1 mod. 2 & Def. $1 M<1.2 M_{\odot}$ \\
\hline Planet-hosts: $\sigma$ whole comp. & $0.031 \pm 0.017$ & $0.030 \pm 0.017$ & 0.031 & $0.031 \pm 0.018$ & $0.011 \pm 0.020$ \\
$\sigma$ M-rich comp. & $0.029 \pm 0.018$ & $0.027 \pm 0.018$ & 0.028 & $0.028 \pm 0.017$ & $0.009 \pm 0.020$ \\
Whole comparison & $0.004 \pm 0.004$ & $0.004 \pm 0.004$ & 0.004 & $0.004 \pm 0.004$ & $0.003 \pm 0.004$ \\
M-Rich comparison & $0.020 \pm 0.011$ & $0.019 \pm 0.012$ & 0.020 & $0.012 \pm 0.007$ & $0.013 \pm 0.011$ \\
Solar comparison & $0.003 \pm 0.005$ & $0.003 \pm 0.004$ & 0.003 & $0.002 \pm 0.003$ & $0.003 \pm 0.005$ \\
\hline
\end{tabular}



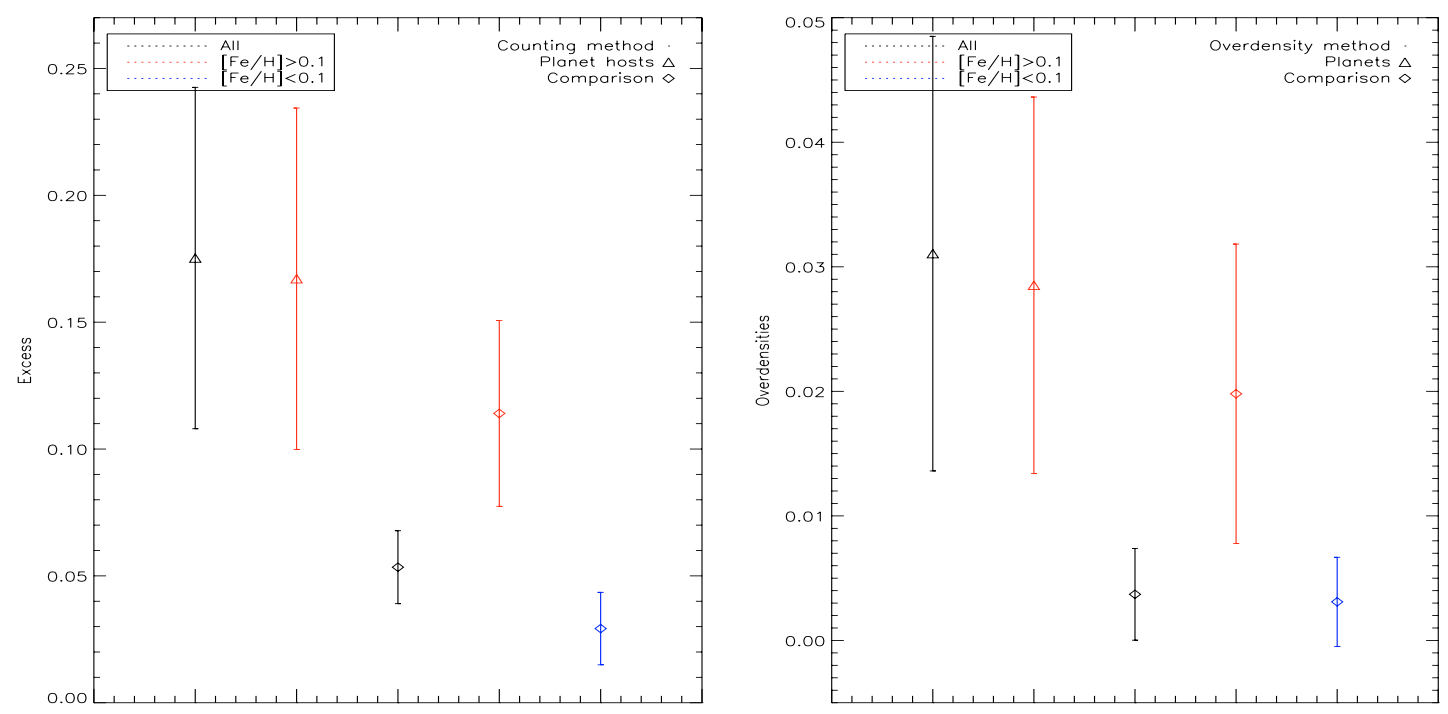

Fig. 11. Results from the "counting" (left panel) and the "overdensity" methods (right panel). Black, red and blue diamonds represent the whole comparison sample, and the metal-rich and solar subsamples, respectively. Black and red triangles indicate planet-host stars, adopting a Gaussian distribution with $\sigma$ equal to the velocity dispersion of the whole comparison sample, and of the metal-rich subsample, respectively. The error bars show Poissonian errors for the "counting" method (left panel), and uncertainties derived by the bootstrap method for the "overdensity" analysis (right panel).

field density to the Hyades overdensity. In the same table we also included the same results for different cases:

- Def. 2: adopting the kinematics parameters of the stream ellipsoids by Famaey et al. (2005b);

- Def. 1 mod. 1 : adopting our kinematic parameters, but without subtracting the averaged field density;

- Def. 1 mod. 2: adopting our kinematic parameters, but adopting $[\mathrm{Fe} / \mathrm{H}]=0.05$ as the metallicity cutoff between the metal-rich and solar comparison subsamples;

- Def. $1 M<1.2 M_{\odot}$ : only the targets with $M<1.2 M_{\odot}$ were included in the analysis in order to check the effect of age.

The errors have been estimated by the bootstrap method, where the estimate of standard error is the standard deviation of the bootstrap replications.

The excess values obtained from the "counting" method (see Table 3, Definition 1), and the overdensities obtained from the "overdensity" analysis (see Table 4, Def. 1), and their corresponding uncertainties, are represented in Fig. 11. The black and red triangles represent the results for planet-host stars, corrected with a Gaussian distribution with $\sigma$ equal to the velocity dispersion of the whole comparison sample and of the metal-rich subsample, respectively.

The "overdensity" analysis (see Table 4, Def. 1) leads to results similar to the "counting" method (see Fig. 11). On the one hand, the comparison metal-rich subsample shows a more significant overdensity in Hyades with respect to the whole comparison sample, agreeing with previous studies. On the other hand, the behaviour of planet-host stars is consistent with what we observe for the metal-rich CORALIE "single" stars, whereas it does not seem to reproduce the kinematic characteristic of the whole comparison sample. In particular, stars with planets present an overdensity in Hyades even more evident than the one found in the metal-rich comparison subsample. The planethost overdensity varies from the value for the comparison sample by $1.5 \sigma$.

No differences emerge if we adopt the stream parameters by Famaey et al. (2005a, see Table 4, Def. 2), or if we do not subtract the average density of the field (see Table 4, Def. 1 mod. 1$)$. For the case adopting $[\mathrm{Fe} / \mathrm{H}]=0.05$ as the metallicity cutoff (see Table 4, Def. 1 mod. 2), there are no significant changes in the results, except for the overdensity of the metalrich comparison subsample, which slightly diminishes. In all the cases, the planet-host stars are much denser in the Hyades stream with respect to the whole comparison sample, with a significance of $1.5 \sigma$.

If younger stars are excluded by considering only targets with $M<1.2 M_{\odot}$, the results for the group of stars with planets deviate from the previous ones (see Table 4 , Def. $1 M<1.2 M_{\odot}$ ). In this case, planet-host stars show a kinematic behaviour almost indistinguishable from the metal-rich comparison subsample, with an equivalent overdensity in the Hyades. Younger planethost stars might be thus responsible for the outstanding excess of stars with planets with respect to the rest.

This raises a question concerning the possible effects of age on the kinematic conduct of the different groups studied. The small number of targets with $M>1.2 M_{\odot}$ does not allow us to study the behaviour of the younger tail independently, so no conclusive argument can be added to discard this possibility. All we can say is that, roughly, a higher percentage (around 50\%) of younger stars is found among planet-host targets with Hyades stream kinematics than among comparison stars of the Hyades stream.

However, the ratio of younger stars in the planet-host group is the same as that found in the metal-rich comparison subsample: 29 younger to 115 older metal-rich comparison targets, and 11 younger to 50 older planet-host stars. This permits us to be more confident that the results are not due to a larger percentage of younger dwarfs in the planet-host sample than in the other groups. The age effect for planet-host targets seems to be strictly related to their membership of the Hyades stream. Moreover, this seems to confirm that planet-host stars behave similarly to the metal-rich population of the solar neighbourhood. It thus represents a further argument in favour of a primordial origin of the metal excess found in stars with giant planets. It cannot be discarded that the clumpiness of young planet-host stars in the Hyades stream might be due to a more efficient planet 

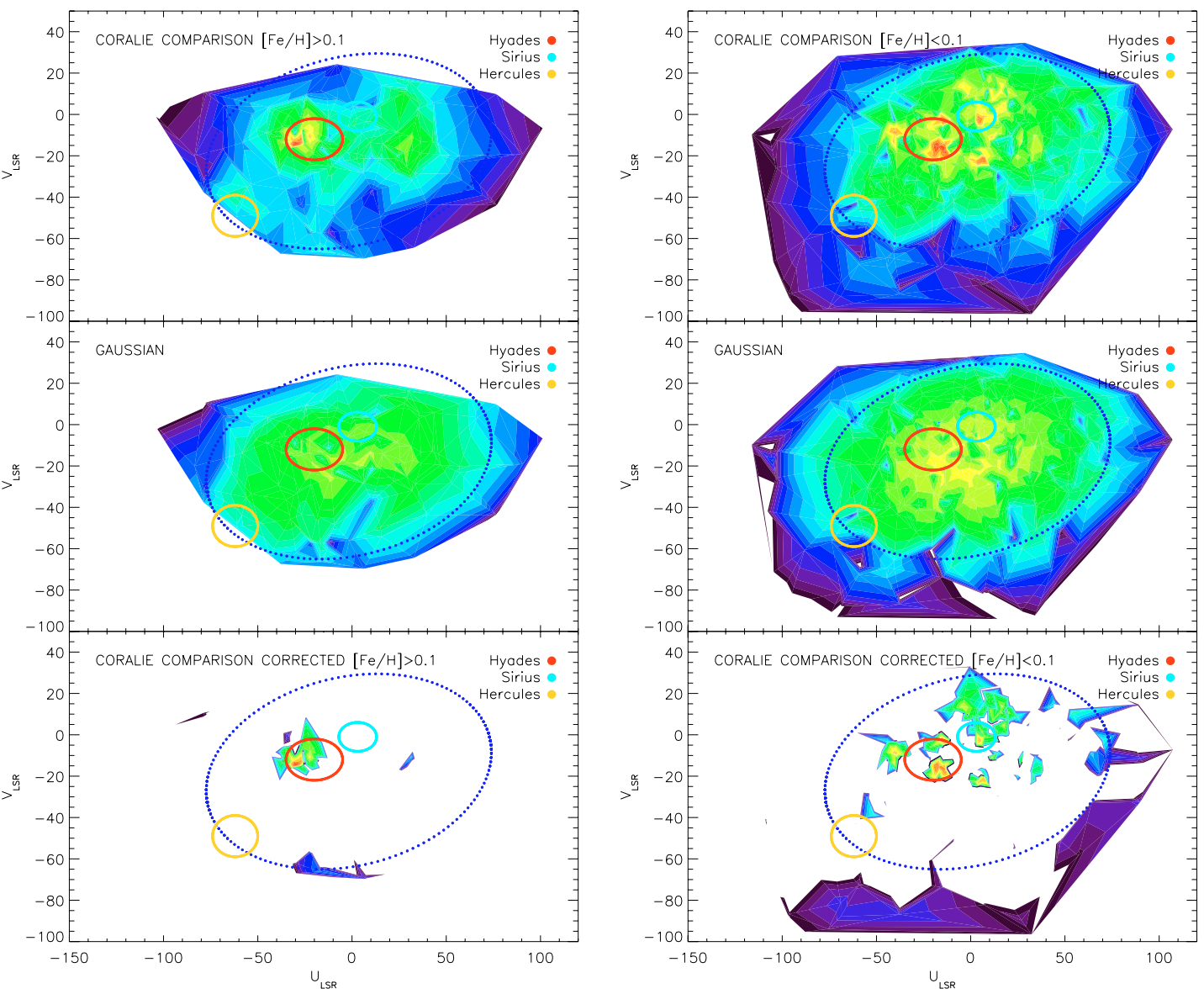

Fig. 12. Density distributions of Galactic space velocities projected on the $(V, U)$ plane, for the metal-rich (left panel) and solar (right panel) comparison subsamples. The uncorrected density distribution, the substracted Gaussian distribution and the corrected density distribution are presented in the upper, central and lower panels, respectively. The field ellipsoid is overplotted with the blue line, while the Hyades, Sirius and Hercules ellipsoids are indicated by the red, blue green and yellow lines.

formation in the primordial Hyades cluster, progenitor of a significant fraction of young members of the Hyades stream.

All our results suggest that planet host stars follow the kinematic conduct of the metal-rich comparison subsample, showing an overdensity in the Hyades stream with respect to the whole comparison group. Furthermore, the younger planet-host targets might present a more significant clumpiness in the Hyades stream that would result in a further overdensity in the Hyades stream, exceeding that found in the metal-rich comparison subsample. However, many more targets are needed to confirm this latter suggestion.

This supports the "primordial" hypothesis, according to which the metal-rich nature of planet-host stars would be due to the high metal content of the protoplanetary clouds from which the systems formed. Moreover, it can also be understood in the scenario proposed as a possible origin of the dynamical streams (see Famaey et al. 2005b, and references therein). According to this hypothesis, the kinematic members of the Hyades stream would have formed in Galactic inner regions, where the intergalactic medium is more metal-rich. Their orbits would have been successively perturbed by spiral waves, causing radial migration towards the solar neighbourhood.

The fact that James et al. (2006) did not find any metalrich population in a sample of three nearby young star forming regions could support the scenario in which most metal-rich field stars come from inner regions in the Galaxy. Moreover, Chiappini (2006, in preparation) found that Galactic chemical evolutionary models, which reproduce most of the abundance trend peculiarities for thin and thick disc populations, cannot explain the existence of the most metal-rich stars of the solar vicinity (at $\sim 8 \mathrm{kpc}$ ). However, those objects are consistent if the same models are computed at inner Galactic radii $(\sim 4 \mathrm{kpc})$.

Dehnen (2000) proposed the Outer Lindblad Resonance (OLR) as the mechanism reproducing the bimodality of the local velocity distribution, identified as the Hercules stream. In this model, closed orbits inside the OLR would move slightly outwards, while those outside the OLR would move inwards, producing two stellar streams. Since the simulations show that the radius of the OLR of the Galactic bar lies inside the solar circle, the stellar velocity distribution observed for late-type stars in the solar neighborhood would be affected by the OLR. These results were confirmed by further simulations by Mühlbauer \& Dehnen (2003). This is the first example of a non-axysimmetric origin for a stream and has opened up new possibilities to relate the other streams with other non-axysimmetric perturbations.

Several simulations have studied the effects of nonaxysimmetric perturbations, in particular of transient spiral waves, on the galactic disc (e.g. Sellwood \& Binney 2002; Bissantz et al. 2003; Chakrabarty 2004; De Simone et al. 2004). They found that spiral waves can cause radial migration near the corotation radius. According to Sellwood \& Binney's model, stars just inside corotation would swap places with those outside, and both groups just would exchange places. In this scenario, Famaey et al. (2005a) interpreted Hyades and Sirius streams as 
the inward- and outward-moving streams of stars on horseshoe orbits that cross rotate. This would easily explain the seemingly peculiar metallicity of these moving groups.

Another interesting feature appears if we compare the density distributions of Galactic space velocities for the metalrich and solar subsamples (see Fig. 12). In the Hyades ellipsoid, the peaks corresponding to the overdensities of the two groups are displaced: the overdensity of the metal-rich subsample (in the left panel) is shifted to lower $U$ values, whereas the solar subsample (right panel) shows a peak shifted towards higher $U$ velocities. Also, the Sirius overdensity completely disappears when considering only metal-rich targets (see right panel). This suggests that the members of the Sirius stream have solar and sub-solar metallicities. Therefore, it is in good agreement with the scenario proposed by Famaey et al. (2005a), according to which the Sirius stream would correspond to the inward-moving counterpart of the Hyades stream.

In this framework, the larger overdensity of planet-host stars observed in the Hyades stream would suggest that at inner galactic radii, in environments more metal-rich than the solar neighborhood, systems with giant planets form more easily. This strongly supports the primordial origin proposed to explain the metal excess observed in stars harbouring giant planets (Santos et al. 2000, 2001). Current models of planet formation by core accretion are in agreement with this observational constraint, and find that metal-rich systems favour the formation of massive planets (e.g. Ida \& Lin 2005; Benz et al. 2006). The combination of this effect with the detection bias towards more massive planets enables models to reproduce the observed correlation between stellar metallicity and the likelihood of harbouring planets (Benz et al. 2006).

\section{Concluding remarks}

This is the first study carried out on a volume-limited sample of stars with and without exoplanets aimed at investigating the comparative kinematics of planet-host stars and their possible relation to the dynamical streams of the solar neighbourhood. We have compared the group of stars harbouring planets with the rest of the CORALIE sample, distinguishing subgroups of metallicity and age. This was made possible by the precise radial velocity measurements and the CCF parameters included in the CORALIE database, and using the HIPPARCOS and Tycho-2 catalogues. For targets with detailed abundance determinations, a complete study of whether they belong to the thick and thin disc populations, and of possible signatures in the abundance trends, has been carried out. The analyses related to the dynamical streams have been carried out using two independent methods that have produced equivalent results.

The main outcomes of this work are the following:

- The CORALIE planet-host target HD 4308 has been found to be a very probable member of the Galactic thick disc, together with another 21 comparison stars; the dependence of the abundance trends on the thin/thick disc populations has been studied for a subset of the CORALIE sample with homogeneous abundance determinations from previous studies.

- No significant differences or peculiar enrichment in $\alpha$-elements are observed in the abundance patterns of the subset of stars with homogeneous abundance determinations from previous studies. However, the small number of thick disc members prevents us from reaching conclusive results.
- The tentative existence of thick disc stars at supersolar metallicities is confirmed by the appearance of one thick member out of 21 with high metallicity (HD 152391 with $[\mathrm{Fe} / \mathrm{H}]=$ $0.03)$.

- The high average metallicity of the Hyades stream reported by previous work is confirmed. Our results are in agreement with the scenario of non-axysimmetric perturbations, such as transient spiral waves, proposed by several results of simulations and observations. According to this model, the Hyades stream would be the outward-moving stream of high-metallicity stars born at inner Galactic radii and then pushed into the solar neighborhood. We have also found a new clue confirming the solar and subsolar average metallicity suggested for the Sirius stream.

- The group of planet-host stars shows a kinematic behaviour much more similar to the metal-rich comparison subsample than to the whole comparison sample, in the sense that the overdensity in the Hyades stream observed for stars with planets is much higher than that observed for the whole comparison group. This conclusion has been reached by the two methods independently and strongly supports a primordial origin for the metal excess observed in stars with giant planets.

- If we interpret our results within the scenario proposed to explain the presence and origin of dynamical streams in the solar neighbourhood, stars with giant planets could have formed more easily at inner Galactic radii in a more metalrich intergalactic medium and then suffered radial displacements due to a non-axysimmetric component of the galactic potential, pushing them into the solar neighbourhood.

This paper presents the results of the first complete analysis on this topic. However, the uncertainties affecting our results are large, due to the intrinsic errors of the kinematics analysis, but also, and mainly, to the inevitably small number of targets with planets in the CORALIE sample. Consequently, substantial improvements will be possible in the near future, when the steadily increasing number of known stars with planetary companions will have risen enough to permit more definite conclusions.

Acknowledgements. This research has made use of the SIMBAD database, operated at CDS, Strasbourg, France. We thank C. Carretero for many fruitful discussions and useful suggestions. Support from Fundação para a Ciência e a Tecnologia (Portugal) to N.C.S. in the form of a scholarship (reference SFRH/BPD/8116/2002) and a grant (reference POCI/CTE-AST/56453/2004) is gratefully acknowledged.

\section{References}

Alcobé, S., \& Cubarsi R. 2005, A\&A, 442, 929

Asiain, R., Figueras, F., Torra, J., \& Chen, B. 1999, A\&A, 341, 427

Barbieri, M., \& Gratton, R. G. 2002, A\&A, 384, 879

Beirão, P., Santos, N. C., Israelian, G., \& Mayor, M. 2005, A\&A, 438, 251

Bensby, T., Feltzing, S., \& Lundström, I. 2003, A\&A, 410, 527

Bensby, T., Feltzing, S., \& Lundström, I. 2004, A\&A, 415, 155

Benz, W., Mordasini, C., Alibert, Y., \& Naef, D. 2006, in Tenth Anniversary of 51 Peg-b: Status of and prospects for hot Jupiter studies, ed. L. Arnold, F. Bouchy, \& C.Moutou, 24

Bissantz, N., Englmaier, P., \& Gerhard, O. 2003, MNRAS, 340, 949

Bodaghee, A., Santos, N. C., Israelian, G., \& Mayor, M. 2003, A\&A, 404, 715

Chakrabarty, D., 2004, MNRAS, 352, 427

Chen, Y. Q., Nissen, P. E., Zhao, G., Zhang, H. W., \& Benoni, T. 2000, A\&AS, 141,491

Chereul, E., \& Grenon, M. 2001, in Dynamics of Star Clusters and the Milky Way, ed. S. Deiters, B. Fuchs, A. Just, R. Spurzem, \& R. Wielen, ASP Conf. Ser., 228, 398

Chereul, E., Creze, M., \& Bienayme, O. 1998, A\&A, 340, 384

Chereul, E., Crézé, M., \& Bienaymé, O. 1999, A\&AS, 135, 5

Chiappini, C. 2006, A\&A, in preparation 
De Simone, R., Wu, X., \& Tremaine, S. 2004, MNRAS, 350, 627

Dehnen, W. 1998, AJ, 115, 2384

Dehnen, W. 1999, ApJ, 524, 35

Dehnen, W. 2000, AJ, 119, 800

Dehnen, W., \& Binney, J. J. 1998, MNRAS, 298, 387

Ecuvillon, A., Israelian, G., Santos, N. C., et al. 2004a, A\&A, 418, 703

Ecuvillon, A., Israelian, G., Santos, N. C., et al. 2004b, A\&A, 426, 619

Ecuvillon, A., Israelian, G., Santos, N. C., Mayor, M., \& Gilli, G. 2006a, A\&A, 449,809

Ecuvillon, A., Israelian, G., Santos, N. C., et al. 2006b, A\&A, 445, 633

Eggen, O. J. 1994, in Galactic and Solar System Optical Astrometry, ed. L.

Morrison, \& G. Gilmore (Cambridge: Cambridge University Press), 191

ESA 1997, The Hipparcos Catalogue, ESA SP-1200

Famaey, B., Jorissen, A., Luri, X., et al. 2005a, A\&A, 430, 165

Famaey, B., Jorissen, A., Luri, X., et al. 2005b, in The Three-Dimensional Universe with Gaia, ed. C. Turon, K. S. O'Flaherty, \& M. A. C. Perryman, ESA SP-576, 129

Feltzing, S., Bensby, T., \& Lundström, I. 2003, A\&A, 397, L1

Fuhrmann, K. 1998, A\&A, 338, 161

Gilli, G., Israelian, G., Ecuvillon, A., Santos, N. C., \& Mayor, M. 2006, A\&A 449, 723

Gonzalez, G., 1997, MNRAS, 285, 403

Gonzalez, G., 1998, A\&A, 334, 221

Gonzalez, G. 1999, MNRAS, 308, 447

Gonzalez, G., Laws, C., Tyagi, S., \& Reddy, B. E. 2001, AJ, 121, 432

Høg, E., Fabricius, C., Makarov, V. V., et al. 2000, A\&A, 355, L27

Hoogerwerf, R., \& Aguilar, L. A. 1999, MNRAS, 306, 394

Ida, S., \& Lin, D. N. C. 2005, ApJ, 626, 1045

Israelian, G., Santos, N. C., Mayor, M., \& Rebolo, R. 2001, Nature, 411, 163

Israelian, G., Santos, N. C., Mayor, M., \& Rebolo, R. 2003, A\&A, 405, 753

Israelian, G., Santos, N. C., Mayor, M., \& Rebolo, R. 2004, A\&A, 414, 601

James, D. J., Melo, C., Santos, N. C., \& Bouvier, J. 2006, A\&A, 446, 971
Lacey, C. G. 1991, in Dynamics of Disc Galaxies, Varberg Castle Sweden, 257 Laughlin, G. 2000, ApJ, 545, 1064

Laws, C., \& Gonzalez, G. 2001, ApJ, 553, 405

Mignard, F. 2000, A\&A, 354, 522

Mishenina, T. V., Soubiran, C., Kovtyukh, V. V., \& Korotin, S. A. 2004, A\&A, 418,551

Mühlbauer, G., \& Dehnen, W. 2003, A\&A, 401, 975

Navarro, J. F., Helmi, A., \& Freeman, K. C. 2004, ApJ, 601, 43

Pietrinferni, A., Cassisi, S., Salaris, M., \& Castelli, F. 2004, ApJ, 612, 168

Pinsonneault, M. H., DePoy, D. L., \& Coffee, M. 2001, ApJ, 556, 59

Pont, F., \& Eyer, L. 2004, MNRAS, 351, 487

Raboud, D., Grenon, M., Martinet, L., Fux, R., \& Udry, S. 1998, A\&A, 335, L61 Reid, I. N. 2002, PASP, 114, 306

Sadakane, K., Ohkubo, M., Takeda, Y., et al. 2002, PASJ, 54, 911

Santos, N. C., Israelian, G., \& Mayor, M. 2000, A\&A, 363, 228

Santos, N. C., Israelian, G., \& Mayor, M. 2001, A\&A, 373, 1019

Santos, N. C., García López, R. J., Israelian, G., et al. 2002a, A\&A, 386, 1028

Santos, N. C., Mayor, M., Naef, D., et al. 2002b, A\&A, 392, 215

Santos, N. C., Israelian, G., Mayor, M., Rebolo, R., \& Udry, S. 2003, A\&A, 398, 363

Santos, N. C., Israelian, G., García López, R. J., et al. 2004a, A\&A, 427, 1085

Santos, N. C., Israelian, G., \& Mayor, M. 2004b, A\&A, 415, 1153

Santos, N. C., Israelian, G., Mayor, M., et al. 2005, A\&A, 437, 1127

Sellwood, J. A., \& Binney, J. J. 2002, MNRAS, 336, 785

Silverman, B. W. 1996, Density Estimation for Statistics and Data Analysis, 6th edn. (London: Chapman \& Hall)

Smith, V. V., Cunha, K., \& Lazzaro, D. 2001, AJ, 121, 3207

Udry, S., Mayor, M., Naef, D., et al. 2000, A\&A, 356, 590

Vauclair, S. 2004, ApJ, 605, 874

Wielen, R. 1977, A\&A, 60, 263

Woolley, R. 1960, in Vistas in Astronomy 3, ed. A. Beer (New York: Pergamon Press), 311 